\title{
Análisis de percepción sobre la nanociencia y la nanotecnología: el caso de la comunidad universitaria de la UNAM*
}

\author{
Gian Carlo Delgado Ramos** y Judith Sarai Peña Jiménez***
}

\begin{abstract}
RESUMEN: Se presenta el análisis de percepción social de la comunidad de la Universidad Nacional Autónoma de México (UNAM) sobre el estado e implicaciones de la nanociencia y nanotecnología a partir de datos arrojados por una encuesta realizada a 500 estudiantes y trabajadores en Ciudad Universitaria (Ciudad de México). Los datos siguen la tendencia de ejercicios similares en otras latitudes. Se concluye con un llamado a fortalecer el apoyo, estímulo y regulación de la ciencia y la tecnología, al tiempo que se procura un trabajo interdisciplinario, así como de periodismo científico, divulgación y otras formas de información, comunicación y diálogo social constructivos y democráticos.

Palabras ClaVE: nanociencia, nanotecnología, percepción social, implicaciones, regulación.
\end{abstract}

ABSTRACT: An analysis on social perception of the community of the National Autonomous University of Mexico regarding current state and implications of nanoscience and nanotechnology is presented. The exercise is a result of a survey carried out at Ciudad Universitaria (Mexico City) on 500 students and workers. Data follows the tendencies of other similar social perception experiences as indicated properly. The text concludes with a call to promote support, stimulate and regulate science ant technology in general, while seeking greater interdisciplinary work, a high level scientific journalism, popularization of science and other forms of informing, communicating and dialoguing with society in a constructive and democratic way.

KEYWORDS: nanoscience, nanotechnology, social perception, implications, regulation.

\section{INTRODUCCIÓN}

Durante los meses de febrero, marzo y abril de 2011 se diseñó una encuesta —véase anexo- para valorar la percepción de la comunidad de la Universidad Nacional Autónoma de México acerca del desarrollo científico tecnológico, el avance de la nanociencia y la nanotecnología y sus implicaciones. La encuesta tuvo un ejercicio piloto que llevó a la necesidad de sumar dos materiales adicionales. Un folleto con las principales definiciones oficiales de lo "nano" y otro folleto extenso de las implicaciones, riesgos y estado actual de la regulación de la nanotecnología. Éste incluyó información proveniente de publicaciones científicas arbitradas sobre las implicaciones positivas y potencialmente negativas en términos económicos, ambientales y a la salud.

Las definiciones ofrecidas fueron las siguientes:

\footnotetext{
* El presente trabajo es parte de los resultados de investigación del proyecto "Laboratorio SocioEconómico de Nanotecnología (LAB-nano)”, convocatoria de apoyo complementario-2009 No. 118244 del Conacyt.

** Economista egresado de la UNAM. Doctor por la Universidad Autónoma de Barcelona. Investigador del programa "El Mundo en el Siglo XXI" del Centro de Investigaciones Interdisciplinarias en Ciencias y Humanidades de la UNAM. Integrante del Sistema Nacional de Investigadores del Conacyt.

*** Estudiante de la licenciatura de sociología. FCPyS, UNAM.
} 
- Royal Society (Reino Unido)

La nanociencia y nanotecnología involucra el estudio y trabajo con la materia a una escala ultra pequeña. Un nanómetro es la millonésima parte de un milímetro y un solo cabello humano tiene alrededor de 80,000 nanómetros de ancho. La nanociencia y nanotecnología abarcan un conjunto de técnicas en lugar de una sola disciplina, extendiéndose por todo el espectro de la ciencia, tocando la medicina, la física, la ingeniería y la química (Royal Society and the Royal Academy of Engineeering, 2004).

- National Nanotechnolgy Initiative (EUA)

La nanociencia y la nanotecnología refiere a la investigación y desarrollo tecnológico a nivel atómico, molecular o macromolecular, en una escala de longitud de aproximadamente 1-100 nanómetros, para proporcionar una comprensión fundamental de los fenómenos y materiales en la nanoescala, y para crear y usar estructuras, dispositivos y sistemas que poseen propiedades y funciones novedosas debido a su pequeño o intermedio tamaño. La investigación y desarrollo en nanotecnología incluye la manipulación bajo el control de las estructuras a nanoescala y su integración en componentes materiales más grandes <www.nano.gov>.

- Clasificación 997 de la USTPO (EUA)

Relativa a la investigación y desarrollo tecnológico a niveles atómicos, moleculares o macromoleculares en escalas de longitud en un rango aproximado de 1 a 100 nanómetros en al menos una dimensión, y que proporciona una comprensión fundamental de los fenómenos y materiales a la nanoescala para crear y usar estructuras, dispositivos y sistemas que tienen propiedades y funciones novedosas debido a su tamaño <www.uspto.gov/web/patents/ classification/uspc977/defs977.htm>.

\section{- Definición de la Oficina Europea de Patentes}

El término nanotecnología cubre entidades con un tamaño geométrico controlado del tamaño de al menos un componente funcional por debajo de los 100 nanómetros en una o más dimensiones susceptibles de hacer disponibles los efectos físicos, químicos o biológicos que son intrínsecos a ese tamaño. Abarca el equipo y los métodos para análisis controlado, manipulación, procesamiento, fabricación o medición con una precisión por debajo de los 100 nanómetros <www.epo.org/news-issues/issues/nanotechnology.html>.

La elaboración del folleto con las definiciones arriba precisadas fue necesaria para que aquellas personas que no sabían qué era la escala nano y por ende la nanociencia y la nanotecnología tuvieran un punto de partida para expresar su opinión acerca de la temática en cuestión sin que fuera informada sobre la diversidad y complejidad de implicaciones, positivas y negativas o de debates existentes. El segundo, se entregó a todos los participantes al final de la encuesta y se les pidió a 1 de cada 10 su opinión después de leerlo, especialmente en cuanto a la utilidad que pudiera tener en relación con su percepción sobre la nanotecnología. Las respuestas fueron variadas pero por lo general se precisó no conocer la información ofrecida en el folleto, encontrándola de utilidad en un grado u otro. Ello con mayor énfasis en el caso de aquellos encuesta- 
dos provenientes de las ciencias sociales y las humanidades. También en algunos casos se expresaron desacuerdos con aspectos puntuales que desde la lectura hecha por los participantes no coincidían con percepciones propias. Incluso fue evidente el desinterés de algunos en tanto "tener" que leer el folleto. Otros puntualizaron conocer la información del mismo.

La encuesta se aplicó a 250 estudiantes y trabajadores del área de las ciencias naturales, exactas y las ingenierías (grupo1), siendo los de mayor peso aquellos de entre 18 a 28 años de edad. También se aplicó a 250 estudiantes y trabajadores del área de ciencias sociales y humanidades (grupo 2), siendo los de mayor peso aquellos de entre 18 a 26 años de edad. El 53\% de los encuestados en del grupo 1 fueron hombres y el $47 \%$ mujeres. En el grupo 2, los porcentajes fueron $34 \%$ y $66 \%$, respectivamente.

La canasta de carreras cubiertas en el grupo 1 fueron en el orden del $40 \%$ a ciencias y $20 \%$ a química, medicina e ingeniería, respectivamente. En el grupo 2 correspondieron con el $22 \%$ a derecho, $21 \%$ a humanidades, $21 \%$ psicología, $21 \%$ ciencias políticas y sociales, $10 \%$ economía y $5 \%$ trabajo social.
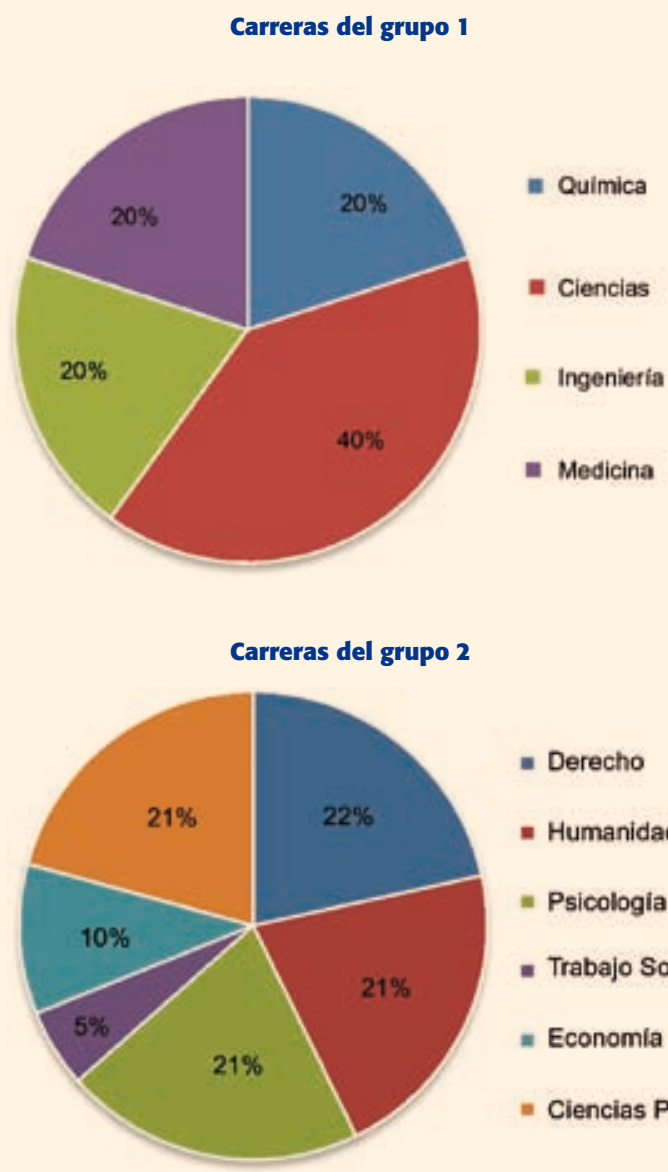

Derecho

Humanidades

Psicologia

Trabajo Social

Economla

Ciencias Politicas y Sociales 


\section{Análisis de resultados del grupo 1}

En el grupo 1 los encuestados se consideran casi en su totalidad, sin importar las diferencias de carrera, como personas interesadas en la ciencia y reportan obtener conocimiento sobre información y novedades tecnológicas por medio de publicaciones electrónicas, artículos en revistas especializadas y Gaceta UNAM <www.dgcs.unam. $\mathrm{mx} /$ gacetaweb>, que resulta un medio muy recurrente para conocer los avances tecnológicos y científicos por parte de la comunidad universitaria, aunque los pertenecientes a medicina e ingeniería, por ejemplo, al contar con una gaceta propia en su facultad, también reportan recurrir a ella como medio de conocimiento, pero en menor grado que los antes mencionados.

Respecto al conocimiento sobre lo que es la nanociencia y la nanotecnología, menos de la mitad de los encuestados respondieron afirmativamente, siendo también una respuesta recurrente el decir que tenían una idea sobre a lo que hacían referencia estos términos, pero no de una manera precisa.

Casi generalizadamente está internalizada la concepción de que la nanociencia y la nanotecnología está vinculada a algo "pequeño" o "microscópico"; una cantidad significativamente pequeña de estudiantes de ciencias duras aluden al término "nano" y describieron la escala de $10^{-9}$, sobre todo en las facultades de ciencias y de ingeniería. No obstante, una idea muy común fue también aquella que relaciona a la nanociencia y nanotecnología con robots.

Los médicos tienden a limitar sus apreciaciones de los probables beneficios de la nanociencia y nanotecnología únicamente dentro del área médica, especialmente aquellos relacionados con la resolución de problemas de índole patológica/sistémica/fisiológica y rara vez aluden a algún otro uso al inicio de la entrevista.

Los demás universitarios tienen una visión un poco más amplia, pero todos coinciden de manera recurrente en la cuestión biomédica e ingenieril de la nanociencia y nanotecnología. Casi ningún encuestado habla sobre avances en el área cosmética, ecológica, alimentaria o textil, salvo casos aislados.

La minoría de los encuestados que respondió conocer algún objeto o servicio derivado de la nanotecnología aludió, en casi todos los casos, a componentes electrónicos/computacionales y aparatos electrodomésticos, pero muy pocos hicieron alusión a cosméticos, medicamentos o textiles.

GRUPO 1. ¿Consideras que los avances de la nanotecnología son seguros para la salud y el ambiente?

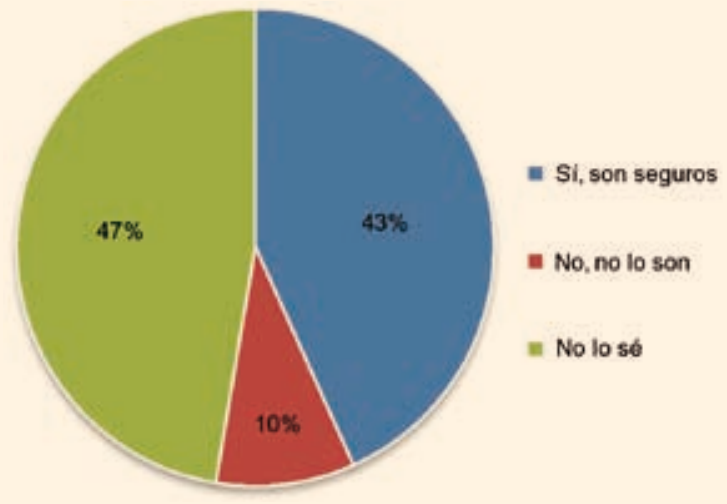


Es de destacar que la enorme mayoría de encuestados del grupo 1 no sabe si la nanotecnología es segura para la salud o el ambiente, pero presentan generalmente opiniones positivas basadas en la creencia de que deben ser seguros por el tamaño que tienen ya que no contaminarían mucho. Se piensa que podrían ser de gran ayuda para curar enfermedades y que serían muy peligrosos en caso de usarse en armas o en objetos "terroristas".

En general, los encuestados del grupo 1 respondieron que es necesaria una regulación de los productos nanotecnológicos ya que muchos coinciden en que se podrían tomar ventajas para los empresarios, como incrementos de precios o monopolización, o ambas. Asimismo, opinan que es importante tal regulación dada la relativa novedad del desarrollo de ésta tecnociencia y por tanto también de sus posibles consecuencias para la salud de los seres humanos. A varios de los encuestados les importó de igual manera el medio ambiente y las probables consecuencias que pueda haber sobre éste.

GRUPO 1. ¿Consideras necesario regular el avance de la nanotecnología y la comercialización de los productos?

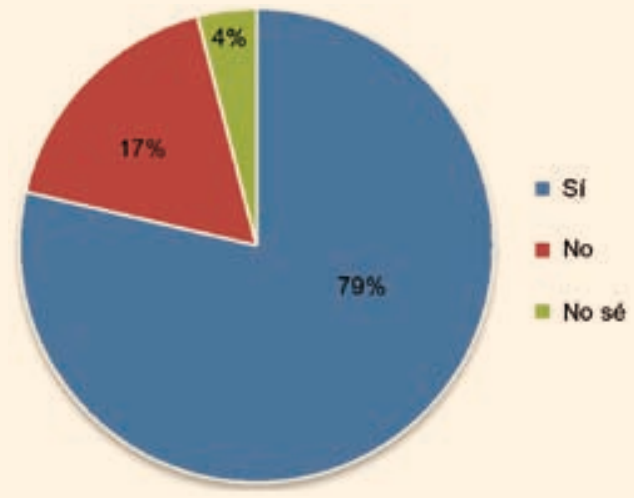

Los encuestados opinan que México cuenta con buenos científicos e investigadores, pero casi el 95\% de los encuestados comparte que el país no invierte lo suficiente en investigación y desarrollo (IyD); que no hay impulso ni apoyo suficiente para la ciencia; que no se apoyará lo suficiente la nanociencia si ni siquiera se impulsa la tecnología "común". También sugieren que, por estos motivos de abandono al campo científico, es que muchos investigadores de excelencia se han ido del país y quizá en este momento estén siendo los protagonistas del desarrollo nanotecnológico en el extranjero donde sí se cuenta con la inversión e infraestructura adecuadas.

Los resultados reflejan que los encuestados del grupo 1 muestran menor confianza en artículos hipotéticamente nanotecnológicos cuando éstos tienen un contacto directo con el ser humano (cuando se comen o se ingieren en forma de medicamentos), pero muestran más confianza en artículos o accesorios que tienen contacto directo con el cuerpo humano; incluso los aprueban a pesar de que pudiera tratarse del mismo nanomaterial. En el caso de los productos de lujo, son pocas las personas que no le otorgan el nivel máximo de confianza, al igual que con los avances en áreas de aplicación "verdes" como las fotoceldas, las cuáles se consideran benéficas para el medio 
ambiente, y en caso de estar hechas con nanotecnología, se asumen como de mayor y mejor funcionamiento.

GRUPO 1. Grado de confianza al utilizar productos que hacen uso de la nanotecnolgía en escala del 0 al 10 donde cero es ausencia de confianza y 10 completamente confiado.

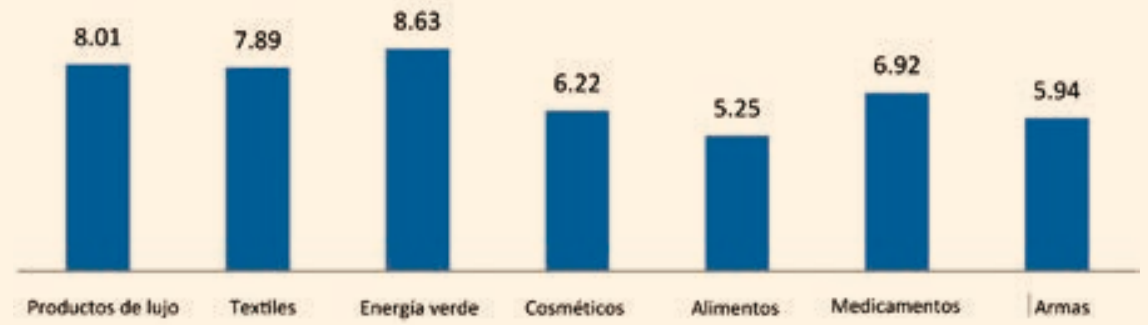

Casi el total de los encuestados opina que los bienes y servicios realizados con nanotecnología deben estar etiquetados indicando sus características para cumplir el derecho de contar con información para que los consumidores decidan sobre la compra y eventual uso de los mismos. A cerca de la regulación de las importaciones, se tiene la opinión mayoritaria de que sí deben ser regulados ya que puede tratarse de productos de baja calidad o de peligro inminente para la salud del ser humano o el ambiente.

GRUPO 1. ¿Crees necesario regular las importaciones de productos nanotecnológicos?

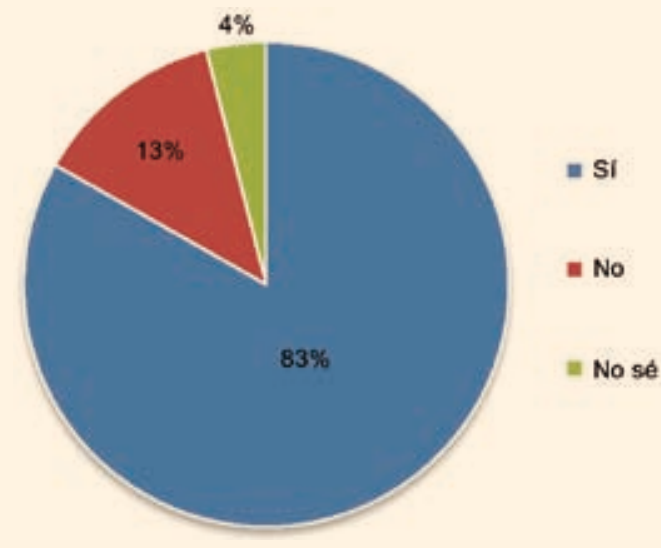

También se indica que debe darse un espacio presencial de mercado, tanto a los avances que entren al país, como a los productos desarrollados en México, ello — se precisa - para evitar que los productos importados coopten todos los nichos de mercado y esto perjudique tanto a las investigaciones nacionales como a los científicos que las realizan. 


\section{Análisis de resultados del grupo 2}

A diferencia de los encuestados del grupo 1, en el grupo 2 el 24\% dijo no estar interesado en la ciencia, mientras que un $76 \%$ dice estarlo (la pregunta no precisa qué tipo de ciencia, lo que confirma que un gran porcentaje de los universitarios de las ciencias sociales y las humanidades asumen automáticamente el concepto de "ciencia" a las ciencias naturales y exactas exclusivamente). Lo anterior se refleja en los porcentajes de reconocimiento sobre el conocimiento de una definición de nanotecnología y nanociencia, que es afirmativo sólo en un 34\%, siendo casi equivalente este porcentaje al de afirmación de conocimiento sobre la existencia de productos nanotecnológicos, que es de $30 \%$. Cifra que difiere poco de los datos del grupo 1, que son de un $33 \%$ y $28 \%$, respectivamente.

Más de la mitad de los estudiantes encuestados respondieron (58\%) que no saben si la nanotecnología y nanociencia son seguras. Esto comprueba que independientemente del área profesional de formación, existe un gran desconocimiento - y no rechazo- sobre este frente tecnológico y sus implicaciones.

GRUPO 2. ¿Consideras que los avances de la nanotecnología son seguros para la salud y el ambiente?

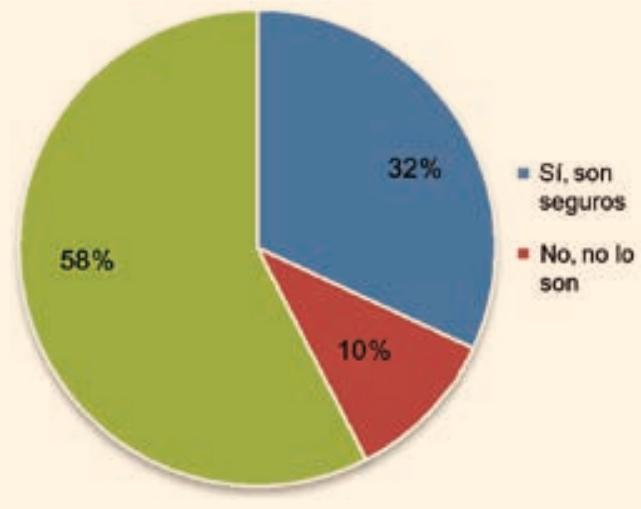

Los resultados reflejan que sólo un $10 \%$ en ambos grupos considera que la nanotecnología es insegura, mientras que un 43\% del grupo 1 y un 32\% del grupo 2 consideran precisamente lo contrario. De notarse es que ambas respuestas, negativas y positivas, parten de grados de conocimiento sobre el tema opuestos $\mathrm{y}$, sin embargo, coinciden en su percepción sobre la cuestión de la seguridad del avance nanotecnológico. Y es que es de recordarse que dos terceras partes del grupo 1 dijo saber qué es nanociencia y nanotecnología mientras que esa misma cantidad del grupo 2 dijo no saberlo.

Los encuestados que afirmaron como segura a la nanotecnología, asociaron de modo muy generalizado sus respuestas a un pensamiento de que "si lo aprueba o lo promueve la ciencia, es seguro". También respondieron que debe ser segura dado que se está trabajando en la mejora y la promoción de su desarrollo para beneficio de la humanidad. Las respuestas reflejan un conocimiento superfluo de la historia y naturaleza de la ciencia y la tecnología, así como una fuerte influencia del paradigma 
normal de la ciencia (en términos kuhnianos [Kuhn, 1971]) acerca de la neutralidad, veracidad y certeza incuestionable de la ciencia, misma que es puesta en duda por la filosofía y la sociología de la ciencia y la tecnología desde hace casi cuatro décadas. Tales disciplinas y en especial los estudios de ciencia, tecnología y sociedad (o STS, por sus siglas en Inglés) y los que se insertan en lo que se ha calificado como ciencia posnormal. Funtowicz, Silvio y Strand, Roger (2007), Gibbons et al., (1997), Ravetz R. Jerome (1971), Ravetz, Jerome y Silvio Funtowicz (1990 y 2000), Strand, Roger y Deborah Oughton (2009), Wynne, Brian (1992) dan cuenta de múltiples casos que corroboran que lo anterior expuesto por los encuestados no necesariamente es así. Ello sobre todo cuando la ciencia y la tecnología se industrializan y corporativizan cada vez más (lo que se ha llegado a calificar como Big Science (Galison, 1992)), así como cuando se observa una fuerte militarización del quehacer científico, sobre todo en Estados Unidos - y que se ha calificado como economía de Pentágono (Melman, 1972)). Por tanto, la necesidad de una formación de carácter interdisciplinario se observa como algo crecientemente deseable.

Ahora bien, en el grupo 2 existe también prácticamente un concenso cuando se habla de la regulación de la nanotecnología, ya que el $87 \%$ de los encuestados dicen que tal regulación debe existir, siendo la respuesta más recurrente que es necesario dado que aún no se conocen las consecuencias reales y tangibles de la nanociencia, por lo que debe existir una exhaustiva regulación para controlar hasta dónde podrían llegar a afectar las innovaciones nanotecnológicas. El dato coincide con los del grupo 1, cuestión que sugiere que independientemente de la formación profesional o del grado de conocimiento sobre la nanotecnología, los usuarios o consumidores siempre prefieren un esquema de regulación que de autorregulación.

GRUPO 2. ¿Consideras necesario regular el avance de la nanotecnología y la comercialización de los productos?

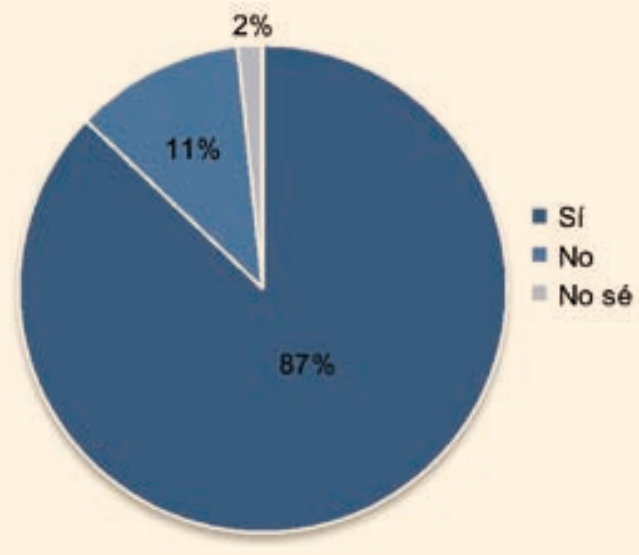

El 72\% de los encuestados en el grupo 1 dice que México no está posibilitado para innovar en nanociencia y nanotecnología y opinan que esto se debe a que, al igual que respondieron los estudiantes de ciencias naturales, no existe suficiente apoyo económico ni inversión adecuada por parte del Estado respecto a la ciencia, ello a pesar de 
que existe tanto buen capital humano como conocimiento en aplicación de la ciencia. Del total de encuestados (considerando ambos grupos), dos terceras partes coinciden en que no hay condiciones ni apoyo suficiente al avance y desarrollo de la ciencia y la tecnología en el país. La percepción coincide con el balance que hizo la Academia Mexicana de Ciencias en el marco del encuentro La Ciencia en México, Zona de Desastre, convocado y celebrado por ésa entidad el 17 de junio de 2009.

Respecto a la confianza que los encuestados respondieron tener en bienes y servicios nanotecnológicos, los números no fueron muy dispares a los del grupo 1 , siendo la menor confianza a las aplicaciones en alimentos, con un promedio de 4.46 en una escala del cero al diez. Le siguen el rubro de las armas, con 4.48, los cosméticos con 5.65 y los medicamentos con 5.96, manteniendo un nivel mayor de confianza los productos que podríamos señalar como "de uso externo" como textiles, con 7.52, los artículos de lujo, con 7.69 y manteniéndose a la cabeza, la producción de energía verde con el promedio más alto, de 8.11 .

GRUPO 2. Grado de confianza al utilizar productos que hacen uso de la nanotecnología en una escala del 0 al 10 donde cero es ausencia de confianza y 10 completamente confiado.

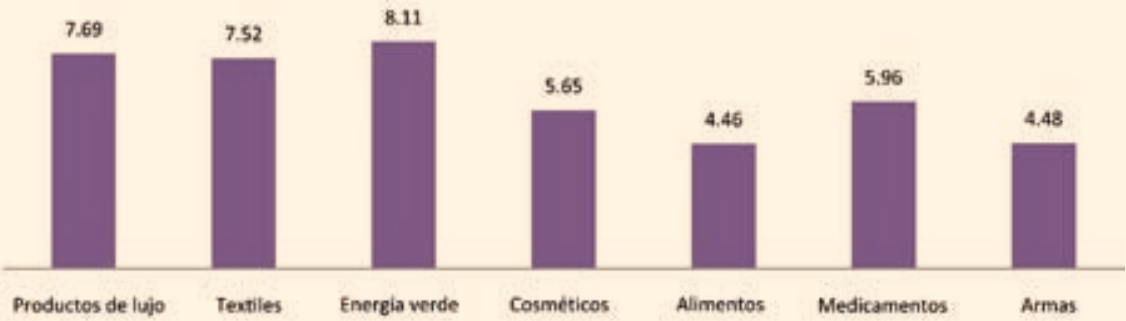

La importancia de etiquetar los bienes y servicios "nano" es del orden del $92 \%$ en el grupo 2, teniendo como principal motivación la de la necesidad de informar al consumidor para que tome sus propias desiciones. De notarse es que algunas respuestas en la Escuela Nacional de Trabajo Social, aludieron a la poca importancia del etiquetado dado que consideraron que "la gente no lo leerá, ya que sólo les importaría el precio, no la información sobre el contenido o modo de producción del producto". En ninguna de las demás facultades se encontraron señalamientos alusivos a tal perspectiva. Por el contrario, el concenso casi total de ambos grupos es por la apuesta al etiquetado de productos nano.

Por último, para el $88 \%$ de los encuestados del grupo 2 es importante la regulación de la nanociencia y los productos nanotecnológicos, siendo la opinión generalizada que, de haber un control legal, podrían prevenirse aspectos riesgosos de los productos, incluyendo los importados; se obligaría a cumplir estándares de calidad; y se controlarían los productos "pirata".

También, al igual que algunos encuestados del grupo 1, en el grupo 2 hubo opiniones que subrayaban la necesidad depromover y favorecer el mercado nacional mexicano frente a productos extranjeros que pudieran ocasionar desventajas para México en términos principalmente económicos. 
¿Crees necesario regular las importaciones de productos nanotecnológicos?

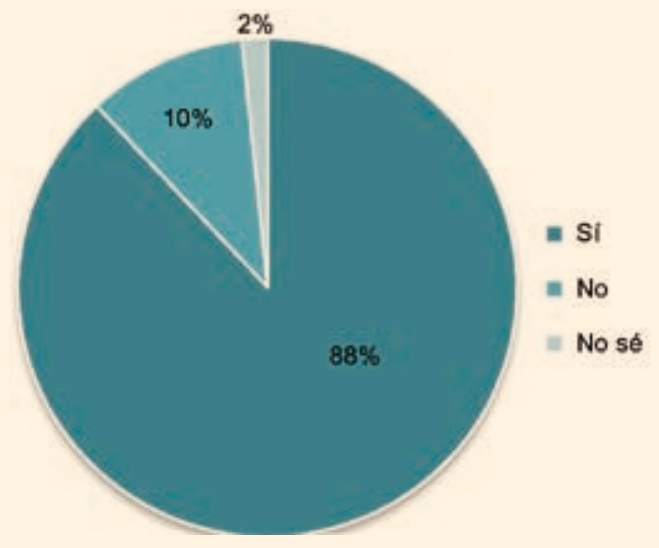

\section{REFLEXIONES finALES}

Los datos arrojados por la encuesta coniciden con algunos indicadores generados en otras latitudes, como los del EuroBarómetro de 2006 (Gaskell et al., 2006) que ofrecen un balance de la percepción en torno a la biotecnología y la nanotecnología en Europa. Asimismo, coincide con las percepciones identificadas en 2010 en las investigaciones del Parlamento del Reino Unido (House of Lords, 2010) en el sentido de que la mayor desconfianza existente en el público de ese país es alrededor del uso de la nanotecnología en productos de contacto directo como los alimentos, siendo de mayor aceptación las aplicaciones medioambientales.

De notarse es que, en efecto, el universo encuestado tiene un sesgo importante: se trata de población con un nivel educativo y de acceso a la información mucho más elevado que el grueso de la población nacional, misma que $8.4 \%$ es analfabeta; $14.4 \%$ no cuenta con educación primaria y $21.2 \%$ no tiene acceso a la educación secundaria. Así, considerando los datos anteriores, es claro que existe mucho por hacer en materia de educación, desarrollo científico y tecnológico, incluyendo, por supuesto, no sólo apoyo, estímulos y regulaciones gubernamentales de mediano y largo plazo, sino también actividades de divulgación, periodismo científico y diálogo social abierto, constructivo, informado e interdisciplinario. Se trata de un ejercicio en el que será necesario ir afinando conceptos para la comunicación adecuada entre los diversos actores, desde los más básicos sobre qué se entiende por ciencia y tecnología o tecnociencia; pasando por cómo se evalúan y se definen las prioridades nacionales, hasta qué se asume cuando se habla de riesgos, peligros, incertidumbre y regulación. Esto y más está por supuesto abierto a discusión, definición, análisis y aterrizaje concreto en políticas públicas y otro tipo de medidas y acciones que se consideren útiles y adecuadas en tanto la realidad específica de cada país. 


\section{AgradeCIMIENTOS:}

Especial agradecimiento por su apoyo en la aplicación de encuestas, vaciado y organización de datos a los siguientes estudiantes de la Facultad de Ciencias Políticas y Sociales de la UNAM: José Manuel Mata Méndez, Maritza Islas Vargas, Liliana Morán Rodríguez, Carolina Bustamante Cruz, Patricia Marisol Rentería Juárez, Valentina Sarmiento Cruz, Sara Susana del Real Pérez, Leslie Cristina Campos Chávez, Emiliano Castillo Jara y Diana Lilia Trevilla Espinal.

\section{Biblografía}

Funtowicz, Silvio y Strand, Roger (2007). “De la demostración experta al diálogo participativo". Revista CTS, España, abril, no. 8, vol. 3: 77-113.

Galison, Peter (ed.) (1992). Big science. The growth of large scale research. Stanford University Press, Stanford.

Gaskell, George et al. (2006). Europeans and biotechnology in 2005: Patterns and trends. Eurobarometer 64.3. Comisión Europea. Disponible en: <http://ec.europa.eu/research/press/2006/pdf/pr1906_eb_64_3_final_report-may2006_en.pdf>.

Gibbons et al. (1997). La nueva producción del conocimiento. Barcelona: Pomares-Corredor. España.

House of Lords (2010). Nanotechnologies and food. Vol. I: Report. The Stationery Office Limited. Londres, Reino Unido. Enero. Disponible en: <www.publications.parliament. uk/pa/ld200910/ldselect/ldsctech/22/22i.pdf>.

Kuhn, Thomas (1971). La estructura de las revoluciones científicas. México: Fondo de Cultura Económica.

Melman, Symour (1972). Capitalismo de Pentágono. La economía política de la guerra. México: Siglo XXI.

Ravetz R. Jerome (1971). Scientific Knowledge and its Social Problems. Nueva York: Oxford University Press,

Ravetz, Jerome y Silvio Funtowicz (2000). La ciencia posnormal. Ciencia con la gente. Barcelona: Icaria.

Ravetz, Jerome y Silvio Funtowicz (1990) Uncertainty and Quality in Science Policy. Londres: Kluwer Academic.

Royal Society and the Royal Academy of Engineeering (2004). Nanoscience and nanotechnologies: Opportunities and uncertainties. Londres, Reino Unido.

Strand, Roger y Deborah Oughton (2009). Risk and uncertainty as a research ethics challenge. National Committees for Research Ethics in Norway, publicación no. 9, Noruega.

Wynne, Brian (1992). "Uncertainty and Environmental Learning-preconceiving Science and Policy in the Preventive Paradigm". Global Environmental Change 2, no. 2: 111-127. 


\section{ANEXO \\ FORMATO DE ENCUESTA}

EDAD:

SEXO:

FACULTAD:

\section{CARRERA:}

1. ¿Te consideras una persona interesada en la ciencia?
a) Sí
b) No

2. ¿Por qué medio te informas de cuestiones científicas? En caso de varios medios, ordenar de forma prioritaria.
a ) Revistas
b) Periódicos
c) Gaceta UNAM
d) Publicaciones electrónicas
e) Televisión
f) Radio
g) Otra- ¿Cuál?

3. ¿Sabes qué es la nanociencia y la nanotecnología?
a) $\mathrm{Si}^{*}$
b) No

* ¿Qué es?

4. ¿Conoces, usas o has visto algún producto derivado de la nanotecnología a la venta en tiendas?
a) Sí* *iDónde?
b) No ${ }^{*}$ Cuál(es)?

5. ¿Consideras que los avances de la nanotecnología son seguros para la salud y el ambiente?
a) Sí, son seguros
b) No, no lo son
c) No lo sé ¿Por qué?

6. ¿Consideras necesario regular el avance de la nanotecnología y la comercialización de los productos? ¿Por qué? 
7. ¿Crees que el nivel científico y tecnológico de México es suficiente para poder innovar en nanotecnología?
a) Sí
b) No

Por qué:

8. ¿Cuál es el grado de confianza que tendrías al utilizar productos abajo indicados en caso de que hicieran uso de la nanotecnología? Indica tu respuesta en una escala del 0 al 10 donde cero es ausencia de confianza y 10 completamente confiado.

\begin{tabular}{|l|l|}
\hline Productos de lujo & \\
\hline Textiles & \\
\hline Producción de energía verde (por ejemplo, fotoceldas) & \\
\hline Cosméticos & \\
\hline Alimentos & \\
\hline Medicamentos y drogas de uso farmacológico & \\
\hline Armas & \\
\hline
\end{tabular}

10. ¿Crees que se deberían etiquetar los productos nanotecnológicos?
a) Sí
b) No

¿Por qué?

11. ¿Crees necesario regular las importaciones de productos nanotecnológicos?
a) Sí*
b) No
* ¿Por qué? 